\title{
ON THE THEORY OF THE CLUSTER SETS OF ANALYTIC FUNCTIONS
}

By

\section{Kiyoshi NOSHIRO}

F. IVERSEN ${ }^{(1)}$, W. GRoss ${ }^{(2)}$ and others ${ }^{(3)}$ have obtained many important results on the cluster sets of analytic functions. The object of this paper is to give some generalizations of and some complements to their results.

First, in $\S 1$, we state an extension of IVERSEN's theorem, using a method for which the writer owes much to J.L. DooB. ${ }^{(4)}$ Next, in $\S 2$, applying NEVANLINNA's theory of harmonic measures ${ }^{(5)}$, we give a more precise form of CARTWRIGHT' ${ }^{(6)}$ extension of IVERSEN's theorem. Finally, in $\S 3$, we enunciate some applications of BEURLING's ${ }^{(7)}$ theorem and give some complements to the principle of maximum modulus.

\section{$\S 1$. An extension of Iversen's theorem.}

1. Suppose that $f(z)$ is a uniform function, meromorphic in a connected domain $D$. Let $z_{0}$ be a point on the boundary $C$ of $D$. We associate with $z_{0}$ the following sets of values:

(1) The cluster set $S_{z_{0}}^{(D)}$. This is the set of all values a such that $\lim _{\nu \rightarrow \infty} f\left(z_{\nu}\right)=\alpha$ where $\left\{z_{\nu}\right\}$ is a sequence of points tending to $z_{0}$

(1) F. IVERSEN : Recherches sur les fonctions inverses des fonctions méromorphes, Thèse de Helsingfors, 1914.

(2) W. Gross : Uber die Singularitäten analytischer Funktionen, Monatshefte für Math. und Phys., Bd. 29 (1918), pp. 1-47.

(3) W. SEIDEL : On the cluster values of analytic functions, Transactions of American Mathematical Society, vol. 34 (1932), pp, 1-21. J. L. DooB: On a theorem of Gross and Iversen, Annals of Mathematics, vol. 33 (1932), pp. 753-757. A. Beurling: Etudes sur un problème de majoration, Thèse de Upsal, 1933. See pp. 100-103. M.L. CARTwRIGHT: On the asymptotic values of functions with a non-enumerable set of singuralities, Journal of London Math. Soc., vol. 11 (1936), pp. 303-305.

(4) Loc. cit.

(5) R. Nevanlinna : Eindeutige analytische Funktionen, 1936. Cf. pp. 106-153.

(6), (7) Loc. cit. 
inside $D$. In other words, $S_{z_{0}}^{(D)}$ is identical with the product of all $\bar{D}_{n}$ where $\bar{D}_{n}$ is the closed cover of the set $D_{n}$ of values taken by $w=f(z)$ inside the common part of $K_{n}$ and $D$, the circle: $\left|z-z_{0}\right|<\frac{1}{n}$ being denoted by $K_{n}$. Hence, it is obvious that $S_{z_{0}}^{(D)}$ is a closed (non empty) set.

(2) The cluster set $S_{z_{0}}^{(C)}$. This is the product $\prod_{n=1}^{\infty} M_{n}$, where $M_{n}$ denotes the closed cover of the sum $\sum_{0<\left|\xi-z_{0}\right|<1 / n} S_{\xi}^{(D)},{ }_{n=1}^{n} \xi$ belonging to $C$. This set is also closed and becomes empty, if and only if $z_{0}$ is an isolated boundary point.

(3) The range of values $R_{z_{0}}^{(D)}$. This is the product of all valuesets $D_{n}$ of $f(z)$ for $K_{n} \cdot D$. Hence, a value $\alpha$ belongs to $R_{z_{0}}^{(D)}$, if and only if, $f(z)$ takes $\alpha$ infinitely often near $z_{0}$ inside $D$. It is clear that $R_{z_{0}}^{(D)}$ is a $G_{\delta}$ set.

(4) The convergence set $\Gamma_{z_{0}}^{(D)}$. Suppose that $z_{0}$ is an accessible boundary point, then there is at least one path (JORDAN arc) lying inside $D$ except the end point at $z_{0} . \quad \Gamma_{z_{0}}^{(D)}$ is the set of all values $\alpha$ such that $f(z) \rightarrow \alpha$ as $z \rightarrow z_{0}$ along such a path. We call such a value $\alpha$ an asymptotic value at $z=z_{0}$. For convenience, we regard $\Gamma_{z_{0}}^{(D)}=0$ when $z_{0}$ is non accessible.

It is obvious that $S_{z_{0}}^{(D)}$ includes $S_{z_{0}}^{(C)}, R_{z_{0}}^{(D)}$ and $\Gamma_{z_{0}}^{(D)}$.

IVERSEN has proved that if $\alpha$ does not belong to $R_{z_{0}}^{(D)}$, where $z_{0}$ is an isolated essential singular point, then $\alpha$ is an asymptotic value at $z_{0}$. The object of this paragraph is to generalize this classical theorem. Let $f(z)$ be a uniform function, meromorphic in $D$, whose two cluster sets are not identical: i.e., $S_{z_{0}}^{(D)}-S_{z_{0}}^{(C)} \neq 0$. Suppose further that a value $\alpha$, lying in $S_{z_{0}}^{(D)}-S_{z_{0}}^{(C)}$, does not belong to $R_{z_{0}}^{(D)}$ : Under these conditions we may assert that $z_{0}$ must be accessible and $\alpha$ is an asymptotic value at $z_{0}$. For proof, we have only to consider the case where $z_{0}$ is non isolated (hence, it is clear that $S_{z_{0}}^{(C)} \neq 0$ ) and $\alpha$ is finite. Since $\alpha$ does not belong to $S_{z_{0}}^{(C)}$, by its definition, there exists a positive number $r_{1}$ such that $\underset{0}{a \bar{\epsilon}} \sum_{0<\left|\xi-z_{0}\right| \leqq r_{1}} S_{\xi}^{(\overline{()}(1)}$ where $\xi$ varies on $C$. Consequently $\alpha$ has a positive distance $\rho_{1}$ from the set $\underset{0<\left|\xi-z_{0}\right| \leqq r_{1}}{S_{(}^{(n)}}$.

(1) " $\alpha \in A$ " and " $\alpha \bar{\epsilon} A$ " mean that " $\alpha$ belongs to $A$ " and " $\alpha$ does not belong to $A$ " respectively. 
By the assumption $\alpha \bar{\epsilon} R_{z_{0}}^{(D)}$, we may also find another positive number $r_{2}$ such that $f(z)-\alpha$ never vanishes for $\left|z-z_{0}\right| \leqq r_{2}$ inside $D$. Let us put $r=\min \left(\frac{r_{1}}{2}, \frac{r_{2}}{2}\right)$. Denote by $K$ and $k$ the circular dise $\left|z-z_{0}\right|<r$ and its circumference $\left|z-z_{0}\right|=r$ respectively. Then $k$ is either contained entirely in $D$ or the common part $k \cdot D$ consists of a finite or an enumerable number of cross-cuts. Consequently the common part $K \cdot D$ is a single connected domain in the former case and contains finite or an enumerable infinity of connected domains $D_{i}(i=0,1,2$, ....) in the latter. We easily see that in either case the modulus of $f(z)-\alpha$ has a positive minimum $\rho_{2}$ on the set $k \cdot D$. Now put $\rho=\min \left(\frac{\rho_{1}}{2}, \frac{\rho_{2}}{2}\right)$. Thus we have obtained two positive numbers $r$ and $\rho$ such that the function $f(z)-\alpha$ does not vanish for $\left|z-z_{0}\right| \leqq r$ inside $D$ and further $\sum_{0<\left|\xi-z_{0}\right| \leqq r} S_{(\Phi)}^{(D)}$ and $\sum_{z \in k \cdot D} f(z)$ both lie outside the circle $(c):|w-\alpha|<\rho$. Remember that $\alpha$ is a cluster value of $f(z)$ at $z_{0}$. Then, there is a sequence of points $z_{\nu}$ inside $D$ such that $z_{\nu} \rightarrow z_{0}$ and $f\left(z_{\nu}\right) \rightarrow \alpha$. Accordingly we may find a point $\zeta_{0}$ in $K \cdot D$, whose image $w_{0}=f\left(\zeta_{0}\right)$ lies within $(c)$; we suppose $\zeta_{0}$ to be contained in the component $D_{0}$ of $K \cdot D$ and denote by $e \epsilon_{0}$ the inverse functional element of $f(z)$ at $w_{0}$. Continuing analytically the element $e_{\xi_{0}}$ inside the circle $\left(c_{0}\right):|w-\alpha|<\left|w_{0}-\alpha\right|$ with algebraic characters, we obtain a branch $z=\phi_{\Delta_{0}}(w)$ of the inverse function of $w=f(z)$; the set of values taken by $z=\phi_{\Delta_{0}}(w)$ in $\left(c_{0}\right)$ is a connected domain $\Delta_{0}, \zeta_{0}$ being its boundary point. Next we investigate the relation between $D_{0}$ and $\Delta_{0}$.

i) $\Delta_{0}$ is contained in $D_{0}$. Suppose that there were a point $z^{*}=\varphi_{\Delta_{0}}\left(w^{*}\right)$ outside $D_{0}$. Then we would have a path $L_{z}$, joining the point $\zeta_{0}$, lying inside $D_{0}$, to $z^{*}$, along which the analytic continuation of $f(z)$ would be possible and whose image by $w=f(z)$ would lie inside $\left(c_{0}\right)$ except the end point $w_{0}$. Obviously $L_{z}$ would cut the boundary of $D_{0}$, while for any point of intersection $\xi$, distinct from $z_{0}, S_{\xi}^{\left(D_{0}\right)}$ lies outside $\left(c_{0}\right)$. Without difficulty, it is seen that there is a contradition.

ii) The closed cover $\overline{\Delta_{0}}$ of $\Delta_{0}$ cannot lie inside $D_{0}$. If it did, $f(z)$ would assume $\alpha$ at least once in $\Delta_{0} .^{(1)}$ This contradicts the fact that $f(z)-\alpha$ never vanishes for $\left|z-z_{0}\right| \leqq r$ inside $D$.

(1) If $\bar{\Delta}_{0}$ were contained in $D_{0}, f(z)-\alpha$ would be a regular function in $\bar{\Delta}_{0}$. having a constant modulus: $|f(z)-\alpha|=\left|w_{0}-\alpha\right|$ on the boundary of $\Delta_{0}$, Hence, we see that $f(z)-\alpha$ would vanish in $\Delta_{0}$, applying the maximum-principle to the function $\psi(z)=(f(z)-\alpha)^{-1}$. 
iii) Accordingly $\Delta_{0}$ and $D_{0}$ must have at least one boundary point in common. However, it is easily seen that there is no common point other than $z_{0}{ }^{(1)}$. Thus, we saw that the domain $\Delta_{0}$, contained in $D_{0}$, has exactly one common boundary point $z_{0}$ with $D_{0}$ and the boundary of $\Delta_{0}$ consists of certain analytic contours $\gamma_{0}$.

Since $f(z)-\alpha$ is regular in the closed domain $\overline{\Delta_{0}}$ except at $z_{0}$, satisfying the relations $0<|f(z)-\alpha|<\left|w_{0}-\alpha\right|$ inside $\Delta_{0}$ and $|f(z)-\alpha|=\left|w_{0}-\alpha\right|$ on $\gamma_{0}$, we can assert, after IVERSEN, that there exists a continuous arc $L_{z}$ contained in $\Delta_{0}$, except the end point $z_{0}$, along which $f(z)$ tends to $\alpha$, as $z$ tends to $z_{0}$. First, $\alpha$ must belong to the cluster set $S_{z_{0}}^{\left(\Delta_{0}\right)}$. To prove this, we consider the function $\psi(z)=\frac{1}{f(z)-\alpha}$, regular in $\Delta_{0}$ and having a constant modulus: $|\psi(z)|=\frac{1}{|f(z)-\alpha|}$ on the boundary $\gamma_{0}$, $z_{0}$ being excluded. Obviously $\psi(z)$ cannot be a bounded function in $\Delta_{0}$, otherwise, by LINDELöF's principle, it would hold that $|f(z)-\alpha| \geqq\left|w_{0}-\alpha\right|$. Accordingly, there is a sequence of points $z_{n}^{\prime}$ inside $\Delta_{0}$ such that $f\left(z_{n}^{\prime}\right) \rightarrow \alpha$ as $z_{n}^{\prime} \rightarrow z_{0}$. Denote by $\left(c_{1}\right)$ the circle: $|w-\alpha|<\frac{\left|w_{0}-\alpha\right|}{2}$ and choose $n$ so large that $w_{n}^{\prime}=f\left(z_{n}^{\prime}\right)$ falls inside $\left(c_{1}\right)$. Next, let us join $\zeta_{0}$ to $z_{n}^{\prime}$ by a simple continuous arc $L_{z}^{(0)}$ within $\Delta_{0}$ except the end point $\zeta_{0}$. Let $L_{w}^{(0)}$ be the image of $L_{z}^{(0)}$ by $w=f(z)$. Clearly $L_{w}^{(1)}$ is a continuous arc inside $\left(c_{0}\right)$ except the end point $w_{0}$, joining $w_{0}$ to $w_{n}^{\prime}$. $L_{w}^{(0)}$ meets at least once at the circumference of $\left(c_{1}\right)$. Denote by $w_{1}$ the first point of intersection from $w_{0}$ along the curve $L_{w}^{(0)}$, and denote by $\zeta_{1}$ the corresponding point; i.e., $w_{1}=f\left(\zeta_{1}\right)$. Thus we see that there is a continuous are $\Lambda_{0}:{\widehat{w_{0} w_{1}}}_{1}$, connecting two circumferences $c_{0}, c_{1}$, of $\left(c_{0}\right),\left(c_{1}\right)$, and lying in the ring $\left(c_{0}\right.$, $c_{1}$ ) except two end points, such that the analytic continuation of $e \zeta_{0}$ along $\Lambda_{0}$ is possible and that the image of $\Lambda_{0}$ by this continuation is a simple arc $\lambda_{0}$, joining $\zeta_{0}$ and $\zeta_{1}$ in $\Delta_{0}$. Next consider the branch $z=\phi_{\Delta_{1}}(w)$ defined inside $\left(c_{1}\right)$ by the element $e_{\zeta_{1}}$, just obtained at $w_{1}$, and let $\Delta_{1}$ be the value set of $z=\phi_{\Delta_{1}}(w)$ in $\left(c_{1}\right)$. Describing the circle $c_{2}:|w-\alpha|=\frac{\left|w_{0}-\alpha\right|}{4}$, whose interior is denoted by $\left(c_{2}\right)$, we see that, by the same reasoning as before, there is a continuous arc

(1) If there were a common boundary point $\xi$, distinct from $z_{0}$, then $S_{\xi}^{\left(\Delta_{0}\right)}$ would be contained in $S_{\xi}^{\left(D_{0}\right)}$, while $S_{\xi}^{\left(\Delta_{0}\right)}$ would lie on the closed cover of $\left(c_{0}\right)$ and $S_{\xi}^{\left(D_{0}\right)}$ entirely outside $\left(c_{0}\right)$. 
$\Lambda_{1}:{\overparen{w_{1} w_{2}}}_{2}$, connecting two circumferences $c_{1}, c_{2}$ and lying between them and having analogous properties to those of $\Lambda_{0}$.

Repeating the same arguments and using analogous notations, for any natural number $n$, we have a continuous arc $A_{n}:{\widetilde{w_{n}}}_{w_{n+1}}$ with the following properties :

1) $\Lambda_{n}$ connects two circles $c_{n}, c_{n+1}$ and lies between them, excluding two end points $w_{n}, w_{n+1}$ where $c_{n}$ is a circle: $|w-\alpha|=\frac{\left|w_{0}-\alpha\right|}{2^{n}}$;

2) Analytic continuation of $e_{\zeta_{n}}$ is possible along $\Lambda_{n}$ and an element $e_{\zeta_{n+1}}$ is obtained at the end point $w_{n+1}$. The image of $\Lambda_{n}$ by this continuation is a simple arc $\lambda_{n}$, joining $\zeta_{n}$ and $\zeta_{n+1}$, inside $\Delta_{n}$.

Since $\lambda_{n}$ falls in $\Delta_{n}-\Delta_{n+1}$, the curve $L_{z}=\sum_{n=0}^{\infty} \lambda_{n}$ is a simple continuous curve in $\Delta_{0}$, and it must converge to $z_{0}$. The last assertion comes from the fact, since we have the inequality $|f(z)-\alpha|>\delta>0$ for $\left|z-z_{0}\right| \geqq \sigma>0$ within $\Delta_{0}$, all $\Delta_{n}$ lie in the circle $\left|z-z_{0}\right|<\sigma$ except a finite number of them. It is obvious that $f(z)$ tends to $\alpha$ as $z$ tends to $z_{0}$ along the curve $L_{z}$.

Thus we have proved the following

Theorem 1. Let $f(z)$ be uniform and meromorphic in an arbitrary domain $D$. Suppose that $\alpha$ is a value belonging to $S_{z_{0}}^{(D)}-S_{z_{0}}^{(C)}$ but not belonging to $R_{z_{0}}^{(D)}$, where $z_{0}$ is a boundary point of $D$. Then $z_{0}$ becomes necessarily accessible and $\alpha$ is an asymptotic value of $f(z)$ at $z_{0}$; that is, a belongs to $\Gamma_{z_{0}}^{(D)}$.

Remark. For the method in our proof we owe much to Dоoв. ${ }^{(1)}$

2. In order to apply the above result, we here propose a lemma concerning $p$-valent analytic functions, obtained easily by means of a method of AHLFORS. Suppose that $f(z)$ is uniform and meromorphic in an arbitrary domain $D$ and that $f(z)$ is at most p-valent in the neighbourhood of a frontier point $z_{0}$, which here we will assume to be non-isolated. Denoted by $K$ the circle: $\left|z-z_{0}\right|<R$, taking $R$ so small that $f(z)$ is at most $p$-valent in the product $K \cdot D$. If we take the image of $K \cdot D$ by $w=f(z)$ on the RIEMANN sphere $\sum$ of radius $\frac{1}{2}$, touching the $w$-plane, its area is obviously at most $p_{\pi}$. Describe a concentric circle $K_{r}:\left|z-z_{0}\right|=r<R$ and denoted by $Q_{r}$ the common part of $K_{r}$ and $D . Q_{r}$ consists of a finite number or an enumerable

(1) He has proved this theorem in the case where $D$ is a Jordan domain. Our theorem is regarded as an extension of his theorem. Cf. DоOB: loc. cit. 
infinity of cross-cuts, except the case in which $K_{r}$ is entirely contained in $D$. In either case, denoting by $L(r)$ the total spherical length of the image of $Q_{r}$ on the $w$-plane, we may obtain the relation $\lim _{r \rightarrow 0} L(r)=0$.

From

$$
L(r)=\int_{Q r} \frac{\left|f^{\prime}(z)\right|}{1+|f(z)|^{2}} r d \theta, \quad\left(z=r e^{i \theta}\right),
$$

applying SCHWARZ's inequality

$$
[L(r)]^{2} \leqq \int_{Q r} r d \theta \int_{Q r} \frac{\left|f^{\prime}(z)\right|^{2}}{\left(1+|f(z)|^{2}\right)^{2}} r d \theta
$$

whence

$$
\frac{[L(r)]^{2}}{2 \pi r} \leqq \int_{Q r} \frac{\left|f^{\prime}(z)\right|^{2}}{\left(1+|f(z)|^{2}\right)^{2}} r d \theta
$$

If we had $\lim _{r \rightarrow 0} L(r)>0$, then there would exist two positive numbers $r_{0}$ and $\delta$ such that $L(r)>\delta>0$ for $r \leqq r_{0}$. Accordingly $\frac{\delta^{2}}{2 \pi} \int_{r}^{r_{0}} \frac{d r}{r} \leqq \int_{r}^{r_{0}} \int_{Q r} \frac{\left|f^{\prime}(z)\right|^{2}}{\left(1+|f(z)|^{2}\right)^{2}} r d r d \theta$, whence we would have $\frac{\delta^{2}}{2 \pi} \log \frac{r_{0}}{r} \leqq p \pi$. Thus we arrive at a contradiction, making $r$ tend to zero.

3. An application of theorem 1 will next be treated, using the just obtained lemma. Let $f(z)$ be uniform and meromorphic in an arbitrary domain $D$ and $z_{0}$ be a non-isolated frontier point of $D$.

There is a problem, not yet solved completely, to find the necessary and sufficient condition that we should have $S_{z_{0}}^{(D)}=S_{z_{0}}^{(C)}$. When $D$ is a JORDAN domain, GRoss ${ }^{(1)}$ has shown that it is sufficient that $R_{z_{0}}^{(D)}$ should be empty, his result being obtained directly from that of BEURLING(2) which we shall state later. Now, we show that for $S_{z_{0}}^{(D)}=S_{z_{0}}^{(C)}$ it is sufficient that $f(z)$ should be at most p-valent near $z_{0}$ inside $D$, without any restriction on the domain $D$. Let $f(z)$ be at most $p$-valent near $z_{0}$. By the lemma, we have a sequence of curves $Q r_{n}$ which satisfy $\lim _{n \rightarrow \infty} L\left(r_{n}\right)=0, r_{n} \rightarrow 0$, denoting by $L(r)$ the spherical total length of

(1) W. Gross : loc. cit.

(2) A. Beurling : loc. cit. 
the image, by $w=f(z)$, of $Q r_{n}$ on the $w$-plane. Any curve $Q r_{n}$ is either a complete circle lying inside $D$ or a system of cross-cuts. Suppose, first, that there are an infinite number of complete circles $Q r_{\nu}$. If we had $S_{z_{0}}^{(D)}+S_{z_{0}}^{(C)}$, then $S_{z_{0}}^{(D)}-S_{z_{0}}^{(C)}$ would contain at least two points $\alpha$ and $\beta, \alpha \neq \beta$, since, in this case, $S_{z_{0}}^{(D)}$ is a continuum and $z_{0}$ is non-isolated. Consequently, by theorem 1, there would exist two paths $L_{\alpha}$ and $L_{\beta}$, inside $D$ and reaching $z_{0}$, along which $f(z)$ would tend to $\alpha$ and $\beta$ respectively, as $z$ tends to $z_{0}$, for no value of $S_{z_{0}}^{(D)}$ belongs to $R_{z_{0}}^{(D)}$. Since $L_{\alpha}$ and $L_{\beta}$ intersect with all circumferences $Q r_{\nu}$, excluding a fininite number, $\alpha$ and $\beta$ would coincide with each other, by the assumption $\lim _{n \rightarrow \infty} L\left(r_{n}\right)=0$. This is a contradiction.

Now consider the other case in which we have a subsequence $\left\{Q r_{\nu}\right\}$, each member being a system of cross-cuts. Again suppose that we had $S_{z_{0}}^{(D)} \neq S_{z_{0}}^{(C)}$. Then denoting by $\alpha$ any value of $S_{z_{0}}^{(D)}-S_{z_{0}}^{(C)}$, we would have a path $L_{\alpha}$, along which $f(z)$ would tend to $\alpha$ when $z$ tends to $z_{0}$. The curve $L_{\alpha}$ would meet at all $Q r_{v}$ except a finite number of them. Denote by $z_{\nu}$ the first point of intersection along $L_{\alpha}$ from $z_{0}$ and by $q_{\nu}$ the cross-cut, containing $z_{\nu}$ in its interior and by $\zeta_{\nu}^{\prime}$ and $\zeta_{\nu}^{\prime \prime}$ the two end points of $q_{v}$. It is obvious that the image of $q_{v}$ by $w=f(z)$ connects two cluster sets $S_{\left.\xi_{v}^{\prime}\right)}^{(P)}$ and $S_{\left.\xi_{v}^{\prime}\right)}^{(D)}$, its spherical length tending to zero as $\nu \rightarrow \infty$. Consequently the value $\alpha$, written in the form $\alpha=\lim _{\nu \rightarrow \infty} f\left(z_{v}\right)$, would belong to $S_{z_{0}}^{(C)}$, since $S_{z_{0}}^{(C)}$ is a closed set. This contradicts the assumption of $\alpha$.

Theorem 2. Suppose that $f(z)$ is uniform and meromorphic in an arbitrary domain $D$ and let $z_{0}$ be a non-isolated boundary point. Suppose further that $f(z)$ is at most p-valent in the neighbourhood of $z_{0}$. Then we have the relation $S_{z_{0}}^{(D)}=S_{z_{0}}^{(C)}$.

Remark. From this theorem follows at once, a result obtained recently by U. MINAMI ${ }^{(1)}$, which is stated in the following form : Let $f(z)$ be a uniform function, holomorphic in $D$, such that with each boundary point $\zeta$, excluding a point $z_{0}$, we can associate a circle, with centre $\zeta$, in which $|f(z)| \leqq m+\varepsilon$, for any positive number $\varepsilon$. Then we have $|f(z)| \leqq m$ throughout inside $D$, provided that $f(z)$ is univalent near $z_{0}, z_{0}$ being a non-isolated boundary point.

(1) U. Minami : An extension of Phragmén-Lindelöf's theorem, Proc. Imp. Acad. Tokyo, vol. 13 (1937), pp. 241-243. 


\section{$\S 2$. A precision of Cartwright's theorem.}

1. Recently CARTWRIGHT ${ }^{(1)}$ has extended IVERSEN's theorem in a distinct way: Let $f(z)$ be uniform and meromorphic in $D$ except a closed set $E$, completely contained in $D$, of essential singularities. Suppose that $E$ is of logarithmic measure zero. If a value $\alpha$ does not belong to $R_{z_{0}}^{(D-E)}, z_{0}$ being a point on $E, \alpha$ is either an asymptotic value at $z_{0}$ or there is a sequence of essential singular points $\zeta_{n}$ tending to $z_{0}$ such that, for every $n, \alpha$ is an asymptotic value at $\zeta_{n}$. Here we give a precise form of her theorem, in virtue of NEvANLINNA's theory of harmonic measures ${ }^{(2)}$. Indeed, in her theorem we can suppose $E$ to be of absolute harmonic measure zero. It is known that, if a set $E$, assumed to be closed, is of logarithmic measure zero, then it is also of harmonic measure zero, its converse being not necessarily true ; moreover, if $E$ is of harmonic measure zero, its linear measure is also zero and $E$ becomes pointwise, that is, $E$ does not contain any continuum as its component.

To prove our assertion we enunciate two lemmas:

Lemma 1. (An extension of WEIERSTRASS' theorem.) Let $f(z)$ be uniform and meromorphic in $D$ except a closed set $E$, lying completely inside $D$, of essential singularities. Let $z_{0}$ be any point of $E$. Then the set $S_{z_{0}}^{(D-E)}$ contains all values, provided that $E$ is of absolute harmonic measure zero.

Lemma 2. (An extension of LINDELöF's principle.) Let $D$ be an arbitrary domain and let $E$ be a closed set of harmonic measure zero, consisting of boundary points. Suppose that $f(z)$ is a uniform, regular function in $D$ which satisfies $|f(z)| \leqq M$ inside $D$, and lim $|f(z)| \leqq m(m \leqq M)$ for any boundary point, not belonging to $E$. Then we have the relation $|f(z)| \leqq m$ throughout in $D$.

Both lemmas may be obtained readily from NevanLINNA's theorems $^{(3)}$ stated in the form of harmonic functions. For instance, we will here consider lemma 1 . Obviously it is sufficient to show that $f(z)$ is not bounded near $z_{0}$. We can draw a circle $K:\left|z-z_{0}\right|=r$ lying entirely inside $D$, for $E$ is of linear measure zero. Consider the real part $u(z)$ of $f(z)$, which is harmonic for $\left|z-z_{0}\right| \leqq r$ inside $D$. and define, by PoIsson's integral, another function $v(z)$ harmonic throughout

(1) M. L. Cartwright : loc. cit.

it R. NevanlinNa : loc. cit. pp. 106-153.

(3) R. Nevanlinna : loc. cit., pages 132 and 134. 
in the circular domain $\left|z-z_{0}\right|<r$ such that $v(z)=u(z)$ on any point on $K$. If $f(z)$ were bounded for $\left|z-z_{0}\right|<r$ inside $D$, then we see that, using NeVANLINNA's result, there would exist the relation $v(z)=u(z)$ throughout for $\left|z-z_{0}\right|<r$ inside $D$. Consequently $f(z)$ would be identical with the analytic function defined by $v(z)$ regular for any point in the circle $\left|z-z_{0}\right|<r$, while $z_{0}$ is an essential singularity of $f(z)$.

2. Now we will give a proof for our assertion. Its proof is entirely similar to that of theorem 1. By hypothesis, there is a positive number $r$, taken to be arbitrarily small, such that the circle $K_{r}:\left|z-z_{0}\right|=r$ lies entirely inside $D^{\prime}=D-E$, as $E$ is of linear measure zero, and such that $f(z)-\alpha$ never vanishes for $\left|z-z_{0}\right| \leqq r$ inside $D^{\prime}$. Consequently we can find a positive number $\delta$, satisfying $|f(z)-\alpha|>\delta>0$ on $K_{r}$. By lemma 1 , there exists a sequence of points $z_{n}$ tending to $z_{0}$ in $D^{\prime}$ such that $f\left(z_{n}\right) \rightarrow \alpha$ as $n \rightarrow \infty$. Denoting by (c) the circular disc: $|w-a|<\delta$, we may find a point $\zeta_{0}$ in $\left|z-z_{0}\right|<r$ whose image $w_{0}=f\left(\zeta_{0}\right)$ lies inside $(c)$. Continuing, with algebraic characters, the inverse element $e_{\zeta_{0}}$ at $w_{0}$ within $\left(c_{0}\right):|w-\alpha|<$ $\left|w_{0}-\alpha\right|$, we have a branch $z=\phi_{\Delta_{0}}(w)$ of the inverse function of $w=f(z)$. Let us denote by $\Delta_{0}$ the set of values taken by $z=\phi_{\Delta_{0}}(w)$ in $\left(c_{0}\right)$. It is clear that $\Delta_{0}$ is a connected domain, its boundary containing a point $\zeta_{0}$. Denote by $D_{r}^{\prime}$ the part of $D^{\prime}$ lying inside $K_{r}$. By a similar reasoning in the proof of theorem 1 , it is seen that i) $\Delta_{0}$ is included in $D_{r}^{\prime}$; ii) $\Delta_{0}$ and $D_{r}^{\prime}$ must have at least one bounday. point, belonging to $E$, in common, but iii) no point on $K_{r}$ belongs to the boundary of $\Delta_{0}$. Thus the boundary of $\Delta_{0}$ consists of a finite or an enumerable number of analytic contours $\gamma_{0}$ lying inside $D_{r}^{\prime}$ and a closed sabset $E_{0}$ of $E$. Obviously the function $f(z)-\alpha$ has the following properties: $f(z)-\alpha$ is regular in the closed domain $\bar{\Delta}_{0}$, excluding all points belonging to $E_{0}$, not vanishing inside $\Delta_{0}$ and moreover statisfies that $|f(z)-\alpha|<\left|w_{0}-\alpha\right|$ in $\Delta_{0}$ and $|f(z)-\alpha|=\left|w_{0}-\alpha\right|$ on the contours $\gamma_{0}$. Accordingly we see that the function $\psi(z)=\frac{1}{f(z)-\alpha}$, regular in $\Delta_{0}$, is not bounded there. In other words, $\alpha$ belongs to the cluster set $S_{\xi}^{\left(\Delta_{0}\right)}$, selecting a point $\xi$ suitably in $E_{0}$. If $\psi(z)$ were bounded, then, applying NEVANLINNA's extension of LINDELÖF's principle, we would have $|\psi(z)| \leqq\left|\frac{1}{w_{0}-\alpha}\right|$ in $\Delta_{0}$, that is, $|f(z)-\alpha| \geqq\left|w_{0}-\alpha\right|$ in $\Delta_{0}$. Consequently we may find a path $\Lambda_{0}$, joining two circum- 
ferences $c_{0}:|w-\alpha|=\left|w_{0}-\alpha\right|$ and $c_{1}:|w-\alpha|=\frac{\left|w_{0}-\alpha\right|}{2}$ and lying inside the ring $\left(c_{0}, c_{1}\right)$, excluding two end points $w_{0}, w_{1}$ along which the analytic continuation of $e_{\zeta_{0}}$ is possible, an element $e_{\zeta_{1}}$ being obtained at the end point $w_{1}$ on $c_{1}$. Moreover it is possible to select $\Lambda_{0}$ so that its image $\lambda_{0}$ is a simple continuous are inside $\Delta_{0}$ joining two points $\zeta_{0}$ and $\zeta_{1}$. Next, from the just obtained element $e \zeta_{1}$ at $w_{1}$, we may similarly define an analytic function $z=\varphi_{\Delta_{1}}(w)$ inside $\left(c_{1}\right)$, whose value set $\Delta_{1}$ on the $z$-plane is contained in $\Delta_{0}$, the boundaries of $\Delta_{0}$ and $\Delta_{1}$ having a common subset $E_{1}$ of $E_{0}$. We may also find a curve $\Lambda_{1}$ with an analogous property to $\Lambda_{0}$, joining two circumferences $c_{1}:|w-\alpha|=\frac{\left|w_{0}-\alpha\right|}{2}$ and $c_{2}:|w-\alpha|=\frac{\left|w_{0}-\alpha\right|}{2^{2}}$ and lying between them, excluding two end points. Repeating the same aguments (Compare the proof of theorem 1.), we finally obtain a path $L_{w}$ joining $w_{0}$ and $\alpha$ inside $\left(c_{0}\right)$, along which the continution of $e_{\zeta_{0}}$ is possible, except the end point $\alpha$, and whose image $L_{z}$ on the $z$-plane by this continuation $z=\varphi_{L_{w}}(w)$ is a simple curve inside $\Delta_{0}$. We must show that $L_{z}$ tends to a point of $E_{0}$. It is obvious that the cluster set $S_{\alpha}^{\left(L_{w}\right)_{(1)}}$ of $z=\varphi_{L_{w}}(w)$ at $w=\alpha$ is a continuum. Since the continuum $S_{\alpha}^{\left(L_{w}\right)}$ must be contained in a pointwise set $E$, it follows that $S_{\alpha}^{\left(L_{w}\right)}$ contains a single point $z^{*}$ in $E_{0}$. In other words, the image $L_{z}$ converges to $z^{*}$ in $E_{0}$ and it is evident that $f(z)$ tends to $\alpha$ along $L_{z}$, if $z$ tends to $z^{*}$. Our assertion has been proved completely, considering $\boldsymbol{r}$ to be taken as small as we please. Thus we have, as a precision of CARTWRIGHT's theorem,

Theorem 3. Let $f(z)$ be uniform and meromorphic in $D$ except a closed set $E$, completely contained in $D$, of essential singularities. Suppose that $E$ is of harmonic measure zero. If a value a does not belong to $R_{z_{0}}^{(D-E)}$, where $z_{0}$ is a point on $E$, then a is either an asymptotic value at $z_{0}$ or there is a sequence of essential singularities $\zeta_{n}$ tending to $z_{0}$ such that, for every $n, \alpha$ is an asymptotic value at $\zeta_{n}$.

Finally we will give, without proof, a further extension of IVERSEN's theorem containing theorems 1 and 3 as its special cases.

(1) This is the set of values $\beta$ such that there is a sequence of points $w_{\nu}$ tending to $\alpha$ on the path $L_{w}$ which satisfies the relation $\lim _{\nu \rightarrow \infty} \varphi_{L_{w}}(w)=\beta$. 
Theorem $4^{(1)}$. Let: $D$ be an arbitrary domain, $C$ being its boundary, and let $E$ be a closed set, included in $C$, which contains only accessible boundary points and whose absolute harmonic measure is equal to zero. Suppose that $f(z)$ is a uniform function, meromorphic in $D$, having a set $E$ of essential singular points. Denoting by $z_{0}$ any point in $E$, we may define a new cluster set $S_{z_{0}}^{*(C)}$ by the formula $S_{z_{0}}^{*(C)}=\prod_{n=1}^{\infty} \quad \sum_{0<\left|\xi-z_{0}\right|<1 / n} S_{\xi}^{(D)}$ where $\xi$ belongs to the set $C-E^{(2)}$. Supposing further that $S_{z_{0}}^{(D)}-S_{z_{0}}^{*(C)}$ is not a vacuum, if a value $\alpha$, contained in $S_{z_{0}}^{(D)}-S_{z_{0}}^{*(C)}$, does not belong to $R_{z_{0}}^{(D)}$, then $\alpha$ is either an asymptotic value at $z_{0}$ or there exists a sequence of essential singularities $\zeta_{n}$ in $E$ tending to $z_{0}$ such that $\alpha$ is an asymptotic value at each $\zeta_{n}$

\section{$\S 3$. Some applications of Beurling's theorem.}

Complements to the maximum principle.

1. Recently BeurLING ${ }^{(3)}$ has obtained the following important theorem :

Theorem $\mathrm{B}_{1}$. Let $D$ be a connected domain and suppose that $z_{0}$ is a boundary point being regular on the problem of DIRICHLET (4). If $f(z)$ is a uniform function, holomorphic and bounded in the neighbourhood of $z_{0}$ inside $D$, then we get $\varlimsup_{z \rightarrow z_{0}}|f(z)|=\varlimsup_{z^{\prime} \rightarrow z_{0}}\left(\varlimsup_{z \rightarrow z^{\prime}}|f(z)|\right)$, denoting any boundary point by $z^{\prime}$.

He has proved it, applying an important, somewhat complicated lemma and recently S. IRIE ${ }^{(5)}$ has given a direct, elementary proof, imposing a slightly stronger restriction on $z_{0}$. The above theorem is written in the form:

Theorem $\mathbf{B}_{2}$. Under the same condition on $D$ and $z_{0}$ as in theorem $B_{1}$, if $\alpha$ is not contained in $S_{z_{0}}^{(D)}$, then $\rho\left(S_{z_{0}}^{(D)}, \alpha\right)=\rho\left(S_{z_{0}}^{(C)}, \alpha\right)$, where $\rho$

(1) The proof is somewhat long and obtained by means of both methods in theorems 1 and 3.

(2) It is clear that $S_{z_{0}}^{*(C)}$ is also closed and contained in $S_{z_{0}}^{(C)}$ (and so in $S_{z_{0}}^{(D)}$ ).

(3) Beurling : loc. cit. See pp. 100-103.

(4) Denote by $E$ the set of values $r$ such that the circumference $\left|z-z_{0}\right|=r$ $(0 \leqq r \leqq 1)$ contains a point, not belonging to $D$. If $E$ contains a segment $[0, \rho]$. selecting $\rho$ to be sufficiently small, then it is known that $z_{0}$ is regular concerning the problem of DiRIChLET.

(5) IRIE has assumed that $z_{0}$ is an accessible boundary point contained in a boundary-component which has at least two points Cf. S. IRIE: Sur un théorème de M. Beurling, Proc. Imp. Acad. vol. 13 (1937), pp. 244-¿46. 
denotes the spherical distance, supposing that $f(z)$ is uniform and meromorphic in $D$.

For, rotating the RIEMANN sphere $\sum$ by the formula $w^{*}=\frac{1+\bar{\alpha} w}{w-\alpha}$, we may replace $\alpha$ by $\infty$ in the above statement, considering $w^{*}(z)=\frac{1+\bar{\alpha} f}{f-\alpha}$, then theorem $\mathrm{B}_{2}$ is identical with theorem $\mathrm{B}_{1}$.

Next we state two elementary lemmas.

Lemma 1. Let $M$ and $N(M>N)$ be two closed sets, on the $w$-plane, such that, for any exterior point $P$ of $M$, they always satisfy $\rho(M, P)=\rho(N, P)$. Then we have $B(M)<B(N)$ where $B(M)$ and $B(N)$ denote the boundaries of $M$ and of $N$ respectively.

The proof is easy. Denote by $Q$ any point in $B(M)$, then, since $M$ is closed, there is a sequence of exterior points $P_{n}$ of $M$ (and $N$ ) such that $P_{n} \rightarrow Q$. Clearly $\rho(M, Q)=\lim \rho\left(M, P_{n}\right), \rho(N, Q)=\lim \rho\left(N, P_{n}\right)$ and, by hypothesis, $\rho\left(M, P_{n}\right)=\rho\left(N, P_{n}\right)$ for any $n$. Consequently $\rho(N, Q)=\rho(M, Q)=0$, whence follows our assertion.

Its converse holds good. That is, we have

Lemma 2. From the assumption that $B(M)<B(N)$ and $M>N$, it follows that, for any exterior point $P$ of $M$, we have $\rho(M, P)=\rho(N, P)$.

Because we have $\rho(M, P) \leqq \rho(N, P)$ and $\rho(B(M), P) \geqq \rho(B(N), P)$, by hypothesis, while $\rho(B(M), P)=\rho(M, P), \quad \rho(B(N), P)=\rho(N, P)$, whence follows at once $\rho(M, P)=\rho(N, P)$, for any exterior point $P$ of $M$.

- By these lemmas, theorem $B_{2}$ is written in the following form:

Theorem $\mathrm{B}_{3}$. Let $f(z)$ be uniform and meromorphic in $D$. Then we have $B\left(S_{z_{0}}^{(D)}\right)<B\left(S_{z_{0}}^{(C)}\right)$ under the same condition on $D$ and $z_{0}$ as in theorem $B_{1}$.

Clearly theorem $B_{1}, B_{2}$ and $B_{3}$ are equivalent with one other. The complementary set of $S_{z_{0}}^{(C)}$ with respect to the $w$-plane is an open set and so decomposed into a finite or an enumerable number of connected components $\Delta_{i}(i=1,2,3, \ldots)$. Now, as an application of theorem $B_{3}$, we have

Theorem 4. Denote by $\Delta$ any one of $\Delta_{i}(i=1,2,3, \ldots)$. Then either $\Delta$ is entirely included in $S_{z_{0}}^{(D)}$ or $\Delta$ never contains any point in $S_{z_{0}}^{(D)}$

Suppose that $\Delta$ contains a point $\alpha$ in $S_{z_{0}}^{(D)}$. If in $\Delta$ there were a point $\beta$, not belonging to $S_{z_{0}}^{(D)}$, then we would find a boundary point of $S_{z_{0}}^{(D)}$, (of $S_{z_{0}}^{(C)}$ by theorem $B_{3}$ ), on a continuous arc $L$, joining two points 
$\alpha$ and $\beta$ inside $\Delta$, since $\Delta$ is a connected domain. Thus we arrived at a contradiction.

BEURLING's results are regarded as localizations of the maximum principle of analytic functions. Concerning the general principle of maximum modulus some analogous results may be obtained.

Let $f(z)$ be a uniform function meromorphic in an arbitrary domain $D, C$ being its boundary. Suppose that $E$ is a closed set, contained in $C$, whose absolute harmonic measure is zero. Denote by $D$ the set of values taken by $f(z)$ in $D$ and put $S_{C-E}=\sum_{z^{\prime} \in C-E} S_{z^{\prime}}^{(D)}$, i.e., the sum of all $S_{z^{\prime}}^{(D)}$, as $z^{\prime}$ varies in $C-E$.

Then it is obvious that their closed covers $\bar{D}$ and $\bar{S}_{C-E}$ satisfy an inequality $\bar{D}>\bar{S}_{C-E}$. Corresponding to theorem $B_{1}$, we may take NEVANLINNA's extension of LINDELöF's principle which we have used already as lemma 2 in $\S 2$.

Theorem $\mathrm{N}_{1}$. If the function $f(z)$ satisfies that $|f(z)| \leqq M$ in $D$ and $\varlimsup|f(z)| \leqq m(m \leqq M)$ for any boundary point belonging to $C-E$, then $|f(z)| \leqq m$ throughout in $D$.

Theorem $\mathrm{N}_{2}$. Let $f(z)$ be uniform and meromorphic in. $D$, its boundary being denoted by $C$, and let $E$ be a closed set, contained in $C$, whose absolute harmonic measure is zero. Then, for any exterior point $\alpha$ of $\mathcal{D}$, we have $\rho(\bar{D}, \alpha)=\rho\left(\bar{S}_{C-E}, \alpha\right)$.

Under the same condition on $f(z)$ and $E$ as in theorem $\mathrm{N}_{2}$, we obtain the following three theorems :

Theorem $\mathrm{N}_{3} \cdot B(\bar{\Phi})<B\left(\bar{S}_{C-E}\right)$.

Theorem 5. Denote by $\triangle$ any component of the complementary set of $\bar{S}_{C-E}$ with respect to the w-plane. Then $\Delta$ is either entirely included in $\bar{D}$ or does not contain any point in $\bar{D}$.

As a corollary, we get a precise form of Minam's result (1).

Theorem 6. If a connected domain $\Delta^{*}$, containing no point of $S_{C-E}$, includes an exterior point a of $\mathcal{D}$, then $\Delta^{*}$ excludes all points of $\bar{D}$.

Because $\Delta^{*}$ contains no point of $S_{C-E}$ and so $\Delta^{*}$ is contained in a component of the complementary set of $\bar{S}_{C-E}$.

2. Now we suppose that $f(z)$ is uniform and meromorphic in $D$ and $z_{0}$ is an arbitrary boundary point. Let us consider the case in which $S_{z_{0}}^{(D)}$ contains an interior point. Denoting by $\alpha_{0}$ such a point,

(1) U. Minami : Sizyô Danwakwai, No. 116, 1936. 
we may find a circle $K_{0}:\left|w-\alpha_{0}\right|<r_{0}$, whose closed cover $\bar{K}_{0}$ is contained entirely inside $S_{z_{0}}^{(D)}$. Let $k_{n}$ be the common part of $D$ and the circle: $\left|z-z_{0}\right|<\frac{1}{n}(n=1,2,3, \ldots)$. Then there is a point $\zeta_{1}$ inside $k_{1}$ whose image $\alpha_{1}=f\left(\zeta_{1}\right)$ belongs to $K_{0}$, since $c_{0}$ is a cluster value of $f(z)$ at $z_{0}$. Consequently we may find a circle $K_{1}:\left|w-\alpha_{1}\right|<r_{1}$ such that its closed cover $\bar{K}_{1}$ is entirely inside $K_{0}$ all values of $\bar{K}_{1}$ are assumed by $f(z)$ inside $k_{1}$. Repeating the same method, we have a sequence of closed circular discs $\bar{K}_{n}$ with the following properties: $\bar{K}_{0}>\bar{K}_{1} \cdots>\bar{K}_{n}>\cdots$ and every value in $\bar{K}_{n}$ is assumed by $f(z)$ in $k_{n}$. Let $\beta$ be a point in $\prod_{n=1}^{\infty} \bar{K}_{n} \neq 0$, then $\beta$ is assumed by $f(z)$ infinitely often near $z_{0}$. Using this elementary fact, we obtain, by theorem $B_{3}$,

Theorem $7^{(1)}$. Let $f(z)$ be uniform and meromorphic in $D$ and let $z_{0}$ be a boundary point, regular concerning the problem of DIRICHLET, Then the set $R_{z_{0}}^{(D)}$ is everywhere dense in the open set $G=S_{z_{0}}^{(D)}-S_{z_{0}}^{(C)}$, provided that $G$ is not empty. Consequently, we have $S_{z_{0}}^{(D)}=S_{z_{0}}^{(C)}$, if $R_{z_{0}}^{(D)}$ is a vacuum. (It is clear that $R_{z_{0}}^{(D)}$ is so, if $f(z)$ does not take any value infinitely often near $z_{0}$ ).

Remark 1. Our theorem 2 is improved, when $z_{0}$ satisfies the condition of regularity concerning the problem of DIRICHLET. There is a difficult problem. Is it true that BEURLING's property $B\left(S_{z_{0}}^{(D)}\right)<B\left(S_{z_{0}}^{(C)}\right)$ holds without any condition of regularity on $z_{0}$ ?

Remark 2. Suppose that $f(z)$ is uniform and meromorphic in a domain $D$, whose order of connection is finite. Then, for any nonisolated boundary point $z_{0}, S_{z_{0}}^{(D)}-S_{z_{0}}^{(C)}$ is an open set, consisting of a finite or enumerable number of connected domains $\Delta_{i}(i=1,2,3, \ldots)$, each being enclosed by $S_{z_{0}}^{(C)}$, (i.e., any boundary point of each $\Delta_{i}$ lies on $S_{z_{0}}^{(C)}$. We have the following results, which are somewhat related to BEURLING's problem ${ }^{(2)}$.

(i) If $z_{0}$ is not accessible, $R_{z_{0}}^{(D)}$ includes every value of $S_{z_{0}}^{(D)}-S_{z_{0}}^{(C)}$.

(ii) If $z_{0}$ is simply accessible, $R_{z_{0}}^{(D)}$ includes every value of $S_{z_{0}}^{(D)}-$ $S_{z_{0}}^{(C)}$, except at most two such values, and, if there are two such exceptional values, then $R_{z_{0}}^{(D)}$ must coincide with the $w$-plane excluding these two points.

(iii) If $z_{0}$ is $k$-ply accessible, $k$ greater than $1, R_{z_{0}}^{(D)}$ contains every value in $S_{z_{0}}^{(D)}-S_{z_{0}}^{(C)}$ except at most $k$ such values.

(1) This is regarded as an extension of WIERSTRASs' theorem.

(2) A. Beurling : loc. cit. See p. 103. 
(i) is already proved in theorem 1. Let $C_{\nu}(\nu=1,2, \ldots m)$ be all components of the boundary $C$ and suppose that $z_{0}$ lies in $C_{1}$. Suppose that near $z_{0}$ we have $k+1$ distinct exceptional values $\alpha_{\nu}(\nu=1,2, \ldots, k+1)$ in PICARD's sense, contained in $S_{z_{0}}^{(D)}-S_{z_{0}}^{(C)}$ then, by means of theorem 1, there exist $k+1$ paths $L_{\nu}(\nu=1,2, \ldots, k+1)$ inside $D$, excluding the common end point $z_{0}$, along which $f(z)$ tends to $\alpha_{\nu}(\nu=1,2, \ldots, k+1)$, as $z$ tends to $z_{0}$, respectively. Without loss of generality, we may suppose that these paths do not intersect one other except at $z_{0}$. Consequently, if we describe a small circle $K:\left|z-z_{0}\right|=r<\delta$, where $\delta$ is the distance of $\sum_{\nu=2}^{\nu=k} C_{\nu}$ from $z_{0}$, the interior of $K$ is decomposed into $K+1$ angular domains. Since $z_{0}$ is $k$-ply accessible, there must exist at least one angular domain $\Delta$, which never contains any point of continuum $C_{1}$, and so is contained entirely in $D$. Applying LINDELöFIVERSEN's theorem ${ }^{(1)}$, we see that $R_{z_{0}}^{(D)}$ contains all values except at most two. Consequently it follows that $k+1 \leqq 2$, that is, $z_{0}$ must become simply accessible. Thus our assertions (ii) and (iii) have been proved at the same time. In particular, if $D$ is a JoRDAN domain, there holds only the case (ii), and this was also proved by Gross and $\mathrm{DOOB}^{(2)}$.

August 1937.

Mathematical Institute

Hokkaido Imperial University.

(1) F. Iversen : loc. cit. See p. 29 and also cf. G. Julia : Leçons sur les fonctions uniformes, 1924, pp. 95-97.

(2) W. Gross : loc. cit. J. L. DooB : loc. cit. pp. 753-757. 